\title{
Opening the Door to Transgender Care
}

\author{
Edward J. Callahan, Ph.D. \\ Davis School of Medicine, University of California, Oakland, CA, USA.
}

J Gen Intern Med 30(6):706-7

DOI: $10.1007 / \mathrm{s} 11606-015-3255-0$

(c) Society of General Internal Medicine 2015

$\mathrm{T}$ his issue of the Journal of General Internal Medicine showcases an intriguing and uncommon article: "Electronic Health Records and Transgender Patients-Practical Recommendations for the Collection of Gender Identity Data." Readers who currently see no transgender or transsexual (now commonly referred to as "trans") patients may wonder why this paper was selected for publication. While the common belief has been that trans patients needed to be referred to endocrinologists or other specialists, there is now increasing recognition that quality primary care can be provided by general internists and others who are willing to expand their practice by engaging in dialogue with the patient and others committed to caring for trans patients. Another common belief in the past was that trans people were universally committed to full medical and surgical transition. The trans patients seeking care now appear to vary widely on how few or how many transition services they seek. Significant expansion and personalization of trans care is critical in efforts to reduce health disparities for these populations.

The pressing need to reduce health disparities for those with marginalized sexual orientation and/or gender identity was brought to public attention with the publication of a committee report from the Institute of Medicine (IOM; 2011). ${ }^{2}$ That report documented serious health disparities in lesbian, gay, and bisexual (LGB) populations, prompting recognition of the medical profession's ethical responsibility to reduce those disparities. ${ }^{3}$ Ironically, almost fully absent from the IOM report was the "T" in LGBT: trans populations. The near silence on trans health stems from the fact that there were so few data on the health of trans populations. In its report, the IOM committee recommends increased efforts to document the gender identity of patients seen in routine care as well as the gender identity of those responding to surveys. Since less than $0.5 \%$ of the population is reputed to be trans, why is this a critical issue for readers of this journal?

First, while gender identity disorders have not yet been the focus of formal epidemiologic studies, Atkins et al. ${ }^{4}$ contend that clinic-referred samples suggest an increasing prevalence. Reviewing ten years of data, Blosnich et al. ${ }^{5}$ reached the same

Published online March 6, 2015

conclusion: they found that the frequency of gender identity diagnoses in the Veteran's Administration health care system nearly doubled from 2002 through 2011. That remarkable increase is prompting new VA initiatives to enhance care for trans patients, including new programs to reduce trans suicidality. Existing data cannot clarify whether the overall incidence of gender dysphoria has increased or whether the noticeable difference reflects more people becoming open about their gender identity. In addition to more individuals appearing to accept and acknowledge their gender identities, it is likely that the Affordable Care Act has increased access to primary care for many trans patients who had little or no access to care in the past.

Second, there is a strong cultural stigma against trans people that often negatively impacts their health. There is evidence of bullying and violence against trans people that begins in the early years, sometimes even within the family, and continues throughout life; ${ }^{6}$ this violence often extends to trans people being the target of sexual violence. Perhaps most importantly, the cultural rejection of children and adults who exhibit crossgender behavior and/or appearance has permeated the health care environment.

Two recent surveys document common negative experiences of trans people seeking health care. An online survey conducted by Lambda Legal ${ }^{7}$ found that nearly $27 \%$ of trans people surveyed reported that they had been denied the health care they sought because of their gender identity. Further, while $21 \%$ reported verbal abuse by their provider, almost $8 \%$ reported that they had experienced rough or physically abusive treatment by a health care professional. In that survey, trans patients reported far higher levels of mistreatment than LGB patients, even higher than the rates reported by HIVpositive patients. It is unknown how many trans patients have stopped seeking care because of their experience at the hands of primary care providers, but it is clear that there is a need for quality improvement in care for trans patients.

To better understand the health needs of sexual minority patients in California, the state Department of Public Health developed a collaborative online survey that was completed by over 3,000 lesbian, gay, bisexual, transgender, queer, and questioning (LGBTQ) individuals. ${ }^{8}$ The survey results revealed that $75 \%$ of respondents agreed with the statement, "I have experienced emotional difficulties such as stress, anxiety or depression which were directly related to my sexual orientation or gender identity/expression," sowing the seeds for increased risk for a variety of health problems. While $77 \%$ 
of those surveyed reported that they had sought care as a result, only $40 \%$ of those who had sought services reported being "satisfied" or "very satisfied" with the care they received. These results suggest a critical need for enhancing the training of health professionals in order to improve care for those with marginalized sexual orientation, and particularly for those with marginalized gender identity or gender expression.

A major part of the poor experience of trans patients may stem from the fact that health practitioners feel that they lack the necessary skills to provide care for this population. A health professional's sense of competence can be severely challenged when they see patients whose identities are unfamiliar. Education about differences in sexual orientation and gender identity has generally been missing in medical and related training.

A recent survey of medical education school deans found little to no coverage of LGBT health in their curricula. ${ }^{9}$ Many schools devoted just a single hour to covering all LGBT health issues, and that hour often covered only HIV. This curricular deficit has been met with a robust response. The Association of American Medical Colleges (AAMC) released a report produced by Hollenbach et al. ${ }^{10}$ in which a curricular map was presented as a guide for health professionals in building programs to educate trainees in sexual orientation and gender identity diversity. While increasing numbers of schools are beginning to mount such efforts, the impact of improved education will be slow to become manifest in the practice of medicine. In addition, those already in the health care workforce need to build their own skills in working with patients for whom they were not taught to provide care. Ironically, in many schools, much of the momentum in building curricular change relies on the energy of trainees themselves, with only secondary support from faculty and staff.

The positive attitudes of younger trainees suggest that improving care and reducing health disparities is possible, perhaps facilitated by an introduction of sexual orientation and gender identity as demographics in the electronic health record (EHR). ${ }^{11}$ While such advances are helpful, changes in attitude will ultimately need to encompass entire health care institutions, from the front desk to the back office, involving all staff who encounter patients. Uncomfortable and disrespectful interactions with office staff are just as effective in driving away patients as poor treatment by a health care professional. The care experience extends beyond the front desk to every individual the patient encounters. To begin to address this needed shift, the New York City Health and Hospitals Corporation released a video, searchable online, entitled "To Treat Me, You Have to Know Who I Am." This simple and poignant video captures patients whose health care experience and health is impacted by their sexual orientation and/or gender identity as they identify themselves in care.

The article by Deutsch et al. is important because it opens the door for care providers to begin the process of becoming more comfortable and competent with trans patients. The article orients the provider to key issues in the care of trans patients, such as the recognition that it is critical to know, respect, and use the patient's preferred name and title (Ms., Mrs., or Mr.), and to understand that the patient's preferred name may differ from their legal name. Indeed, perhaps the most basic and critical aspect of the relationship between a provider and a trans patient is respect for the patient's gender identity as reflected in their preferred name and in the sensitive use of preferred pronouns.

It takes courage for trans patients to open the door to enter primary care medicine. Are we prepared to welcome them with respect and compassion?

Conflict of Interest: The author has no conflicts of interest on this topic.

Corresponding Author: Edward J. Callahan, Ph.D.; Davis School of MedicineUniversity of California, Oakland, CA, USA (e-mail: ejcallahan@ucdavis.edu).

\section{REFERENCES}

1. Deutsch MB, Buchholz D. Electronic Health Records and Transgender Patients-Practical Recommendations for the Collection of Gender Identity Data. J Gen Int Med [SPI 3148]. January 6.

2. Institute of Medicine. Committee on Lesbian, Gay, Bisexual, and Transgender Health Issues and Research Gaps and Opportunities; Board on Health of Select Populations. The Health of Lesbian, Gay, Bisexual, and Transgender People: Building a Foundation for Better Understanding. Washington, DC: National Academies Press; 2011. http://www.iom.edu/ Reports /2011/The-Health-of-Lesbian-Gay-Bisexual-and-TransgenderPeople.aspx Accessed February 9, 2015

3. Callahan EJ, Hazarian S, Yarborough M, et al. Medicine's ethical obligation to eliminate LGBT health disparities: The associated roles of electronic health records and institutional culture. Hastings Cent Rep. 2014;44(5):S48-S52.

4. Aitken M, Steensman TD, Blanchard $\mathbf{R}$ et al. Evidence for an altered sex ratio in clinic-referred adolescents with gender dysphoria. J Sex Med. 2015. doi:10.1111/jsm. 12817.

5. Blosnich JR, Brown GR, Shipherd JC, et al. Prevalence of gender identity disorder and suicide risk among transgender veterans utilizing Veterans Health Administration Care. Am J Publ Health. 2013;103(10):e27-e32.

6. Stotzer RL. Violence against transgender people: A review of United States data. Aggression and Violent Behavior. 2009;14(3):170-179.

7. Lambda Legal. When Health Care Isn't Caring. Lambda Legal's Survey on Discrimination Against LGBT People and People Living With HIV. 2010; http://www.lambdalegal.org/publications/when-health-care-isnt-caring Accessed February 9, 2015

8. Mikalson P, Pardo S, Green J. (2012). First, Do No Harm: Reducing disparities for lesbian, gay, bisexual, transgender, queer, questioning populations in California. 2014; http://www.cdph.ca.gov/programs/ Documents/LGBTQ_Population_Report.pdf Accessed February 9, 2015

9. Obedin-Maliver J, Goldsmith ES, Stewart L, et al. Lesbian, gay, bisexual, and transgender- related content in undergraduate medical education. JAMA. 2011;306:971-977.

10. Hollenbach $\mathrm{AD}$, Eckstrand KL, Dreger A, eds. Implementing curricular and institutional climate changes to improve health care for individuals who are LGBT, gender nonconforming, or born with DSD: A resource for medical educators. Washington: AAMC; 2014.

11. Callahan EJ, Sitkin N, Ton $\mathbf{H}$, et al. Introducing sexual orientation and gender identity Into the electronic health record: One academic health center's experience. Acad Med. 2015;90(2):154-160. 